\title{
Optical coherence tomography angiography in uveitis
}

Paris Tranos ${ }^{1}$, Evdoxia-Maria Karasavvidou ${ }^{1,2^{*}}$, Olga Gkorou ${ }^{1}$ and Carlos Pavesio ${ }^{3}$

\begin{abstract}
Before the introduction of optical coherence tomography angiography (OCTA) in the early 2000s, dye-based angiography was considered the "gold standard" for the diagnosis and monitoring of ocular inflammation. OCTA is a novel technique, which demonstrates capillary networks based on the amount of light returned from moving blood cells, providing further information on pathophysiological changes in uveitis.

The aim of this review is to describe the basic principles of OCTA and its application to ocular inflammatory disorders. It particularly emphasizes on its contribution not only in the diagnosis and management of the disease but also in the identification of possible complications, comparing it with fundus fluorescein angiography (FFA) and indocyanine green angiography (ICGA). Although the advent of OCTA has remarkably enhanced the assessment of uveitic entities, we highlight the need for further investigation in order to better understand its application to these conditions.
\end{abstract}

Keywords: Optical coherence tomography angiography, Uveitis, Chorioretinal inflammation, Dye-based angiography, Blood flow

\section{Introduction}

In recent years various imaging modalities have emerged facilitating effective investigation, diagnosis and monitoring of ocular disorders [1]. In 2006, Makita et al. first described optical coherence tomography angiography (OCTA), a novel imaging modality which utilizes the advances in OCT technology to provide new insight in retinal microvascular changes, without the requirement of intravenous dye injection. This innovative technique provides highresolution angiographic information that can be objectively correlated to OCT anatomic findings [2-4].

Uveitis is associated with a spectrum of pathologic processes including inflammation, vascular occlusion or leakage, local ischemia, and alteration of cellular mediators. Visually debilitating complications such as macular edema and neovascularization among others may potentially occur. Also, some inflammatory lesions may be difficult to differentiate from a vascular lesion. Early identification and monitoring of these changes may be

\footnotetext{
* Correspondence: evikarassavidou@gmail.com

${ }^{1}$ Vitreoretinal \& Uveitis Department, Ophthalmica Clinic, Vas.Olgas 196 and

Ploutonos, 54655 Thessaloniki, Greece

${ }^{2}$ Department of Ophthalmology, Hippokrateio General Hospital of

Thessaloniki, 49 Konstantinoupoleos Street, 54642 Thessaloniki, Greece

Full list of author information is available at the end of the article
}

critical in the optimal management of patients with uveitis. Recent studies suggest that the use of OCTA, in conjunction with the other imaging modalities, can be advantageous in patients with ocular inflammation, revealing features which may improve our knowledge on pathophysiology and natural course of the disease and guide decision making for the uveitis specialist [3].

In this review, a comprehensive overview of the principles of OCTA and its application to ocular inflammatory disorders has been performed.

\section{Dye-based angiography}

\section{Fundus fluorescein angiography}

Fundus fluorescein angiography (FFA) is an imaging modality based on the intravenous administration of fluorescein dye [5]. This technique is widely used predominantly for the demonstration of retinal vascular abnormalities, especially leakage, macular or optic disc edema and non-perfusion [6]. However, vascular features of the retina may be underestimated due to the limited resolution of FFA and, as an example, choroidal neovascularization $(\mathrm{CNV})$ may not be detected due to masking from coexisting subretinal hemorrhage [7]. Fluorescein molecular size is also an important issue, as small 
molecules leak more quickly and prevent detailed view of the vascular structures [8]. Fundus fluorescein angiography can be associated with mild (nausea, vomiting, sneezing, pruritus) or more severe adverse reactions (syncope, local tissue necrosis, thrombophlebitis, local skin eruptions at the site of injection) [9]. Cardiovascular shock, myocardial infarction, laryngeal edema and bronchospasm have also been reported and are important contributors to the mortality rate [10].

\section{Indocyanine green angiography}

Yannuzzi et al. [11] first described indocyanine green angiography (ICGA), which is usually performed to reveal choroidal involvement in uveitis and identify occult choroidal neovascularization (CNV) [12, 13], polypoidal choroidopathy, and $\mathrm{CNV}$ complicated with subretinal hemorrhage [14]. In comparison with FFA, the emission wavelength of indocyanine green (ICG) is longer; thus, deeper retinal structures can be better visualized. Additionally, a higher proportion of ICG is albumin bound ( 98\%) [15], so the leakage of dye decreases and the signal to noise ratio improves. Consequently, ICGA is more reliable in imaging the choroidal vasculature and choroidal pathology allowing better visualization of the choriocapillaris and choroidal stroma, which are affected in many visually threatening inflammatory conditions. Nevertheless, this method has limitations similar to FFA since presence of subretinal fluid, subretinal hemorrhage or retinal pigment epithelium (RPE) detachment may also obscure CNV or other retinal features [16]. Severe adverse reactions have also been reported during ICGA, especially in subjects with iodine allergies $[15,17]$.

\section{Optical coherence tomography angiography Basic principles}

Optical coherence tomography angiography is an expansion of spectral-domain optical coherence tomography (SD-OCT) [1], providing detailed visualization of functional vasculature of ocular tissue [18].

The basic principle of OCTA relies on the variation in OCT signal produced by the reflectance of light off the surface of moving red blood cells [1, 18]. Structural tissues generate steady signal, while flowing blood cells produce signal that changes over time because of the continuous motion. OCTA scans are repeated over the same area in order to discriminate the moving particles from static tissue [18]. OCT device emits light which can be reflected, refracted, or absorbed [1] and uses two main methods to develop the angiographic contrast: (1) the amplitude decorrelation (or intensity) and (2) the phase variance. These two methods compare the differences between the light reflected in various vessels and the background, aiming to detect significant motion and allowing detailed depiction of retinal and choroidal microvasculature [1].

\section{Limitations and artifacts}

Despite its many advantages over dye-based angiography techniques, OCTA has some technical limitations. The limited view of field $(8 \mathrm{~mm} \times 8 \mathrm{~mm})$ of the currently available devices prevents the wide visualization of the posterior pole and restricts the evaluation of pathology predominantly affecting the peripheral retina. OCTA provides more concise depiction of ocular microcirculation. Nevertheless, it requires accurate segmentation for the identification and quantification of vascular abnormalities of the eye [4].

In line with any other imaging methods, OCTA can be influenced by artifacts which may lead to data misinterpretation.

Media opacities such as cataract, small pupil or defocusing of the light beam have the potential of producing weakened OCT signal and inaccurate flow information. All these shortcomings may be moderated by focusing and centering the OCT beam and by dilating the patients' pupils [4]. In addition, small eye movements, such as changes of fixation, saccadic or movements associated with tremor and breathing, are sources of artifacts that could lead to overestimation of flow signal $[1,4]$. However, eye tracking software and motion correction techniques have become available on commercial OCTA devices, decreasing the defects of OCTA scans [19-21]. Moreover, flowing red blood cells into the vessels of superficial choroidal plexus can cast shadows over the deeper vascular layers [4]. These artifacts result in projection of the same vascular pattern of superficial layers on deeper retinal circulation including the normally avascular layer of photoreceptors. Consequently, detection and quantification of vascular abnormalities in the outer retina may become difficult due to blockage from projection artifacts, which can be misinterpreted [22]. OCTA devices have a threshold for slow flow signal detection; hence, areas with blood flow under this threshold may not be detected and incorrectly maybe characterized as non-perfused [1].

\section{Optical coherence tomography angiography in uveitis}

Optical coherence tomography angiography vs dye-based angiography

Due to its micromolecular properties, fluorescein may easily leak from retinal vessels with the slightest disruption of the blood-retinal barrier. FFA is a very sensitive imaging modality for the detection of retinal vessel inflammation, because even minor inflammation of the retinal vessel wall may result in vascular leakage. This leakage on FFA is extremely useful feature in order to assess the activity of underlying uveitis, both in terms of 
intensity and extent. On the other hand, OCTA is not able to detect leakage, but can depict changes in the vessel density of superficial or deep capillary plexus in vasculitis. Kim et al. [2] identified that the parafoveal capillary density in the superficial retinal plexus was significantly lower in eyes with retinal vasculitis compared with healthy ones. These results suggest that OCTA can potentially be used to quantitatively measure the effects of intraocular inflammation.

Dye-based angiography poorly visualizes the deep capillary plexus (DCP) in contrast to OCTA. Evaluation of deeper vascular components can be useful in some uveitis entities including birdshot retinochoroiditis, which has been shown to be associated with decreased flow density in the DCP [23]. Lower flow density is not necessarily associated with non-perfusion. Leakage of plasma outside the vessel wall in retinal vasculitis results in decrease of flow velocity inside the vessel. However, in active inflammation accompanied by leakage on FFA, retinal circulation can be identified on OCTA scans despite the lower flow velocity.

Dye leakage in posterior uveitis may be helpful for the assessment of the inflammatory activity, but it can be restrictive in the evaluation of adjacent capillary perfusion. In this respect, the use of OCTA has proven advantageous, providing details on microvascular morphology and information about capillary perfusion in both superficial and deep capillary plexus.

FFA is considered the "gold standard" for the detection of any type of CNV [24]. However, even with multimodal imaging, it is very difficult to differentiate active inflammatory lesions from inflammatory CNV, as both have the potential to cause a breakdown in the blood-retina barrier. Usually, inflammatory $\mathrm{CNV}$ is characterized by early hyperfluorescence with late leakage, while inflammation shows early hypofluorescence with late hyperfluorescence on FFA [25]. Not infrequently, this early hyperfluorescence of $\mathrm{CNV}$ may not be obvious due to blockage from inflammatory component or hemorrhage. Moreover, inflammatory lesions may show early hyperfluorescence because of the window defect caused by RPE damage, which may be present adjacent to new active lesions.

ICGA provides better imaging of the choroidal vasculature through the RPE [26] since in contrast to FFA, the lesions are not obstructed by significant choriocapillaris leakage [27]. ICGA leakage results from significant damage to retinal and choroidal stroma vessels, but dye leakage from fenestrated choriocapillaris may be very slow in case of inflammation. Herbort et al. demonstrated the involvement of choriocapillaris and choroidal stroma in the pathophysiology of several inflammatory diseases of the fundus which can cause characteristic findings on ICGA [28].

\section{Microvascular changes in uveitis}

OCTA is the only imaging method that can depict microvascular changes of the superficial and deep capillary plexus in detail, since there is no masking by leakage, pooling or window defects $[29,30]$ (Table 1 ).

The stability of ocular function at the level of neurosensory retina is largely dependent on the integrity of the inner and outer blood-retinal barrier [31, 32]. The human immune system produces inflammation by releasing various inflammatory mediators including prostaglandins, interleukins (e.g., IL-1, IL-2, and IL-6), interferon gamma and tumor necrosis factor alpha (TNF-a). All these mediators contribute to the disruption of the inner blood-retinal barrier, resulting in an influx of fluid from vessels to the intraretinal or subretinal space and formation of extracellular edema.

Recent studies using OCTA showed that uveitic macular edema is associated with changes in the density or morphology of deep capillary plexus (DCP). Their analysis revealed a significantly lower vessel density in DCP in uveitic eyes complicated by macular edema. In addition, ocular inflammation was associated with parafoveal capillary loss in the superficial capillary plexus regardless of the presence of macular edema [2]. Besette et al. [1] identified similar findings including the remodeling of capillaries and the irregularity and/or enlargement of foveal avascular zone (FAZ) in uveitis patients.

Blood flow changes associated with active inflammation are characterized by capillary dropout or loss in the SCP that can be identified as hypo-perfused areas in OCTA. However, apparent areas of non-perfusion may only represent slow flow, considering the inadequacy of the device in detecting slow flow signal [1].

\section{OCTA in anterior uveitis}

Pichi et al. was the first to demonstrate quantitatively the iris vasculature of subjects with anterior uveitis using OCTA. By applying the appropriate settings for iris scanning, they found that inflamed irises had better highlighted microvasculature than the healthy ones, with radial small vessels packed more densely towards the pupillary margin and irregular vessels arranged less densely towards the iris root [1].

In order to quantify the increase in flow and the dilation of vessels which occur in anterior uveitis, Pichi et al. compared the brightness of grayscale OCTA scans before and after uveitis treatment. Their measurements showed that the brightness was significantly increased in severe inflammation than in mild cases. In addition to measuring brightness, they attempted to calculate the vascular volume of inflamed irises. Study data revealed much higher vascular volume in eyes with $4+$ anterior chamber cells, decreasing with improvement of inflammation. Those results based on OCTA measurements 
Table 1 Pathological features detected on different layers of OCTA in various uveitis entities

\begin{tabular}{|c|c|c|c|c|c|}
\hline & $\begin{array}{l}\text { Superficial capillary } \\
\text { plexus }\end{array}$ & Deep capillary plexus & Outer retina & Choriocapillaris & Choroid \\
\hline Retinal vasculitis & $\begin{array}{l}\text {-Decreased flow density } \\
\text {-Enlargement/ Irregularity of FAZ } \\
\text {-Capillary remodeling }\end{array}$ & $\begin{array}{l}\text {-Grayish hypo/non perfused areas } \\
\text {-Elevated, dilated or shunting } \\
\text { perifoveal vessels } \\
\text {-Well delineated flow void areas }\end{array}$ & & & \\
\hline $\begin{array}{l}\text { Birdshot } \\
\text { retinochoroiditis }\end{array}$ & $\begin{array}{l}\text {-Telangiectatic vessels } \\
\text {-Increased intercapillary space in } \\
\text { the perifoveal region }\end{array}$ & & & & \\
\hline $\begin{array}{l}\text { Ocular } \\
\text { toxoplasmosis }\end{array}$ & & $\begin{array}{l}\text { Neovascular network arising from } \\
\text { retinal vasculature with no } \\
\text { contribution of the choroid }\end{array}$ & & & \\
\hline MEWDS & & & & Normal flow & \\
\hline APMPPE & & & & $\begin{array}{l}\text { Flow reduction } \\
\text { and ischemia }\end{array}$ & $\begin{array}{l}\text { Flow reduction and } \\
\text { ischemia }\end{array}$ \\
\hline $\mathrm{PIC} / \mathrm{MCP}$ & & & $\begin{array}{l}\text { Tangled vessels } \\
\text { arising from } \\
\text { choriocapillaris }\end{array}$ & $\begin{array}{l}\text {-Focal flow } \\
\text { reduction (MCP) } \\
\text {-Tangled vessels } \\
\text { extending into } \\
\text { outer retina }\end{array}$ & \\
\hline $\begin{array}{l}\text { Serpiginous } \\
\text { choroiditis }\end{array}$ & & & & $\begin{array}{l}\text { Flow reduction in } \\
\text { areas of active } \\
\text { lesions }\end{array}$ & $\begin{array}{l}\text { Better delineated } \\
\text { vessels in areas of } \\
\text { inactive lesions }\end{array}$ \\
\hline Sarcoidosis & & & & & Flow void areas \\
\hline Tuberculosis & & & & & $\begin{array}{l}\text {-Flow void areas } \\
\text {-Tangled vessels } \\
\text { arising from } \\
\text { choriocapillaris }\end{array}$ \\
\hline VKH & & & & Focal flow void & \\
\hline $\begin{array}{l}\text { Uveitic macular } \\
\text { edema }\end{array}$ & & Decreased capillary density & & & \\
\hline $\begin{array}{l}\text { Inflammatory } \\
\text { CNV }\end{array}$ & & & $\begin{array}{l}\text { Neovascular } \\
\text { network }\end{array}$ & & \\
\hline
\end{tabular}

established a new perspective in the qualitative and quantitative assessment of iris vasculature using OCTA. However, this approach is limited due to some anatomical and technical parameters:

(1) Pupil size needs to be constant as it may affect the morphology of iris vasculature and OCTA results.

(2) OCTA measurements may not be reproducible because of differences in iris pigmentation.

(3) Ocular tissue refraction of OCTA beam can give a false impression of anterior segment physical characteristics.

\section{OCTA in retinal vasculitis}

Retinal vasculitis may be a consequence of various ocular and systemic diseases including systemic lupus erythematosus (SLE), Adamantiades-Behçet disease (ABD), multiple sclerosis (MS), ocular tuberculosis (TB), and sarcoidosis among others. Inflammation of retinal vessels may be a component of almost all types of intermediate and posterior uveitis.
In active vasculitis, fundoscopy reveals focal, multifocal, or diffuse white sheathing of retinal vessels. Perivascular infiltration by inflammatory cells accounts for the pathological vessel sheathing, which is postulated to generate the blurred margins of vessels. Retinal vasculitis may be accompanied by other vascular changes including telangectasias, vascular anastomosis, microaneurysms, macroaneurysms and optic disc or preretinal neovascularization [1].

FFA remains a remarkably useful imaging modality for the diagnosis and monitoring of retinal vasculitis. Fluorescein is able to escape through breaks in blood-retinal barrier, allowing the detection of vascular occlusion or leakage [3]. On the other hand, recent studies have shown that OCTA can demonstrate enlargement and/or irregularity of the foveal avascular zone (FAZ) and capillary remodeling [1], specifically in patients with involvement of the macula.

Khairallah et al. [33] with the aid of OCTA highlighted the presence of grayish nonperfused/hypoperfused areas mostly involving the deep capillary plexus (DCP) in patients with ABD vasculitis. Similar findings have been 
observed in retinal vascular occlusion, diabetic retinopathy and sickle cell retinopathy [34-38]. Deep capillaries are more susceptible to ischemia as they are not directly connected to arterioles. Additional findings included elevated, dilated, or shunting perifoveal capillary vessels and well-delineated black, roundish, or elongated areas devoid of flow. The formation of cystoid spaces displacing the peripheral capillaries might explain the total absence of flow in these areas. However, caution should be taken as retinal atrophic alterations, involving the macula and the retinal nerve fibers, may produce projection artifacts on OCTA, indicating nonperfusion/hypoperfusion of both the superficial and deep capillary plexus, where none exists.

\section{OCTA in retinitis and choroiditis Birdshot retinochoroiditis}

Birdshot retinochoroiditis (BRC) is a rare bilateral chronic posterior uveitis, strongly associated with human leukocyte antigen (HLA) A29 [39]. Clinical picture is characteristic, with multiple scatter, oval, creamy white hypopigmented choroidal lesions, vitritis, retinal vasculitis, and macular edema.

De Carlo et al. [40] performed OCTA on eight eyes with BRC to evaluate the microvascular alterations occurring in the superficial retinal capillary plexus of the posterior pole. Their study data revealed the presence of abnormal telangiectatic vessels and an increase of intercapillary spaces in all affected eyes. They also observed capillary dilatations and loops in seven of the total eight eyes examined.

Twenty-two patients (forty-four eyes) with BRC, which were considered inactive based on clinical examination and imaging with OCT and FFA, were evaluated by Pichi et al. [1]. In their series, foveal avascular zone (FAZ) and the area of capillary nonperfusion were delineated and measured with the use of OCTA at the level of superficial capillary plexus (SCP). Their results showed that subjects with BRC had a larger FAZ area compared with the healthy ones. Another important microvascular change being noticed was the enlargement of perifoveal intercapillary spaces in all eyes with BRC, representing areas of perifoveal ischemia. All these findings could lead to the assumption that chronic BRC induces hypoperfusion of the SCP, resulting in tissue hypoxia and cellular death.

In another study held by Pohlmann et al. 64 eyes of 32 subjects with BRC were classified according to disease activity and duration of the disease. They were evaluated with multimodal imaging which revealed that active BRC was associated with retinal vasculitis and hyperfluorescence of the optic disc on FFA as well as hypofluorescent areas on ICGA. In all eyes, OCTA demonstrated capillary loops and telangiectatic vessels, altered retinal vascular architecture and rarefication of $\mathrm{C}$-scans in retinal layers. However, increased rarefication of $\mathrm{C}$-scans and altered retinal vascular architecture in SCP and DCP were significantly correlated with disease activity [41].

\section{Ocular toxoplasmosis}

Ocular toxoplasmosis is frequently associated with retinal vasculitis, and may also result in serous retinal detachment, occlusive vascular disease, macular edema and $\mathrm{CNV}$. Dye-based angiography is usually performed for the diagnosis of atypical cases and the identification of complications. On FFA, the lesion appears hypofluorescent in the early images followed by gradual leakage from surrounding vessels. In the presence of $\mathrm{CNV}$, a typical progressive leakage over the area of new vessels is demonstrated on FFA. On ICGA, the main lesion of ocular toxoplasmosis is depicted as a hypofluorescent area with multiple satellite hypofluorescent foci, whereas $\mathrm{CNV}$ appears hyperfluorescent [3]. ICGA also shows involvement of the choriocapillary and choroid in acute toxoplasmic retinitis.

Spaide [42] recently described the use of OCTA in order to evaluate an area of $\mathrm{CNV}$ in a patient with ocular toxoplasmosis. Leakage on FFA indicated the presence of $\mathrm{CNV}$ but its precise anatomy could not be identified. OCTA scans showed significant contribution to the neovascular tissue from the retinal vasculature without any participation from the choroid. Hence, it is obvious that we can perform OCTA to assess the origin of abnormal vessels developed not only in toxoplasmosis but also in other inflammatory diseases.

\section{OCT-A in choroiditis (choriocapillaropathies and stromal choroiditis)}

Multiple evanescent white dot syndrome

Multimodal imaging can be extremely useful in patients with multiple evanescent white dot syndrome (MEWDS) and subtle clinical findings. Electroretinography (ERG) demonstrates photoreceptor dysfunction [43], while in fundus autofluorescence (FAF) hyperfluorescent spots are highlighted in the early stages, as the RPE autofluorescence is unmasked due to misalignment of the photoreceptors or metabolic changes affecting RPE [44]. FFA shows early hyperfluorescence of the dots with late staining, while on ICGA more numerous hypofluorescent spots are illustrated than the corresponding seen clinically or on FFA $[45,46]$. On structural en face OCT hyporeflectivity is seen at the level of the RPEphotoreceptor complex that co-localizes with the hypofluorescent spots on ICGA, supporting the hypothesis that the dark areas on ICGA are attributable to focal non-functional RPE cells not absorbing the ICG molecule. OCTA is of great importance in revealing that 
within the corresponding hypofluorescent spots of ICGA, the choriocapillaris and retinal capillary blood flow are completely normal. This reinforces the concept that choriocapillaris may not be involved in this disease and the primary cause of it stands at the level of RPEphotoreceptor complex [1, 23, 47-49]

\section{Acute posterior multifocal placoid pigment epitheliopathy}

FFA demonstrates early hypofluorescence of the acute posterior multifocal placoid pigment epitheliopathy (APMPPE) lesions and late hyperfluorescence due to staining [50-52]. APMPPE has been proposed to be a result of ischemia and acute inflammation of the choriocapillaris, but not of the medium and large sized choroidal vessels, leading to RPE abnormalities [53-55]. This inner choroidal involvement as prominent feature is supported by the ICGA findings of hypofluorescent areas corresponding to the lesions observed on FFA [51, 52, 56, 57]. OCT imaging manifests increased inner choroidal hyporeflectivity or lucency $[52,58]$, ellipsoid zone disruption and sub-RPE drusenoid abnormalities with RPE atrophy ensuing in the course of the disease, all of which co-localize with the primary zone of choroidal hypoperfusion [1, 59]. OCTA imaging confirmed that hypothesis of inner choroidal or choriocapillaris flow reduction, as it illustrates true choriocapillaris flow-void areas that correspond to the ICGA hypofluorescent spots and are of the same or larger size. These findings are not artifacts, since they are not associated with signal attenuation or blockage due to overlying RPE alterations [1, 23, 58-63]. Other studies have also showed progressive recovery of blood flow with reduction of the ill perfused area and evidence of vascular reperfusion $[1,59,60,62]$.

\section{Multifocal choroiditis and panuveitis}

Along with direct damage of the retina and RPE due to inflammation, common vision-threatening complications of multifocal choroiditis and panuveitis (MCP) include inflammatory $\mathrm{CNV}$, cystoid macular edema and subretinal fibrosis [64-67]. In active disease, FFA shows early hypofluorescence due to blockage from the lesions and late hyperfluorescence due to staining, while atrophic scars look hyperfluorescent due to window defect [3, 68, 69]. ICGA illustrates hypofluorescent acute lesions, some of which may not be clinically apparent [26]. On OCT inflammatory lesions are typically presented as homogenous elevations of the RPE that may penetrate into the subretinal space and outer retina $[1,23,66,70,71]$. More heterogeneous subretinal material, occasionally with a sub-RPE component, may indicate the presence of $\mathrm{CNV}[23,66,71,72]$. However, differentiating purely inflammatory lesions from inflammatory CNVs can be potentially difficult, as both lesions may leak on FFA [23, 66]. OCTA has been used to assess vascular alterations and is able to precisely identify $\mathrm{CNV}$ as a lacy vascular network at the level of a normally avascular area of outer retina. Although OCTA cannot accurately define the level of $\mathrm{CNV}$ activity in cases of inflammatory CNV, it is the diagnostic modality of choice since findings of other imaging modalities can be largely equivocal and difficult to interpret $[1,3,23,64,66,73,74]$. Therefore, OCTA findings are crucial and may entirely determine the therapeutic approach, because a combination of immunosuppressive agents with intravitreal anti-VEGF therapy in cases of inflammatory CNV is mandatory, as opposed to monotherapy with immunosuppression in purely inflammatory lesions without CNV. OCTA at the level of choriocapillaris has also shown flow-void areas that correspond to the hypofluorescent areas on ICGA. However, caution should be taken in cases where RPE atrophy is present, which may result in a hyper intense vascular network on OCTA, attributable to the visible Sattler layer's vessels under the atrophic RPE and the undetected signal of the choriocapillaris blood flow [73].

\section{Punctate inner choroidopathy}

The clinical course of punctate inner choroidopathy (PIC) is considered to be benign unless complicated with vision-threatening consequences, which include subretinal fibrosis and CNV [3, 66, 75, 76].

In the acute phase, FFA depicts early hypofluorescent spots with late staining, while $\mathrm{CNV}$ is typically illustrated with a lacy hyperfluorescent pattern followed by late leakage $[3,77,78]$. On ICGA hypofluorescent spots are evident at the level of choriocapillaris [3]. On OCT active lesions are typically presented as elevations of the RPE, which may extend into inner retina, which decrease converting to areas of sub-RPE accumulations or outer retinal disruption when inactive. Hyperreflective heterogeneous subretinal material, accompanied or not with intraretinal fluid, may be indicative of a neovascular membrane $[3,23,66]$. OCTA has been shown to effectively reveal and confirm the presence of a neovascular network of choroidal origin in patients with PIC, even in apparently inactive cases, suggesting an increased risk of recurrence [79] (Fig. 1).

Similar to MCP, OCTA findings can be advantageous in differentiating purely inflammatory lesions from inflammatory $\mathrm{CNV}$, in cases where findings from other imaging modalities are inconclusive. Moreover, it can be useful in monitoring patients with $\mathrm{CNV}$ and its response to therapy $[3,66]$.

\section{Serpiginous choroiditis}

Fundus autofluorescence (FAF) is remarkably useful in evaluating the inflammatory activity of serpiginous 

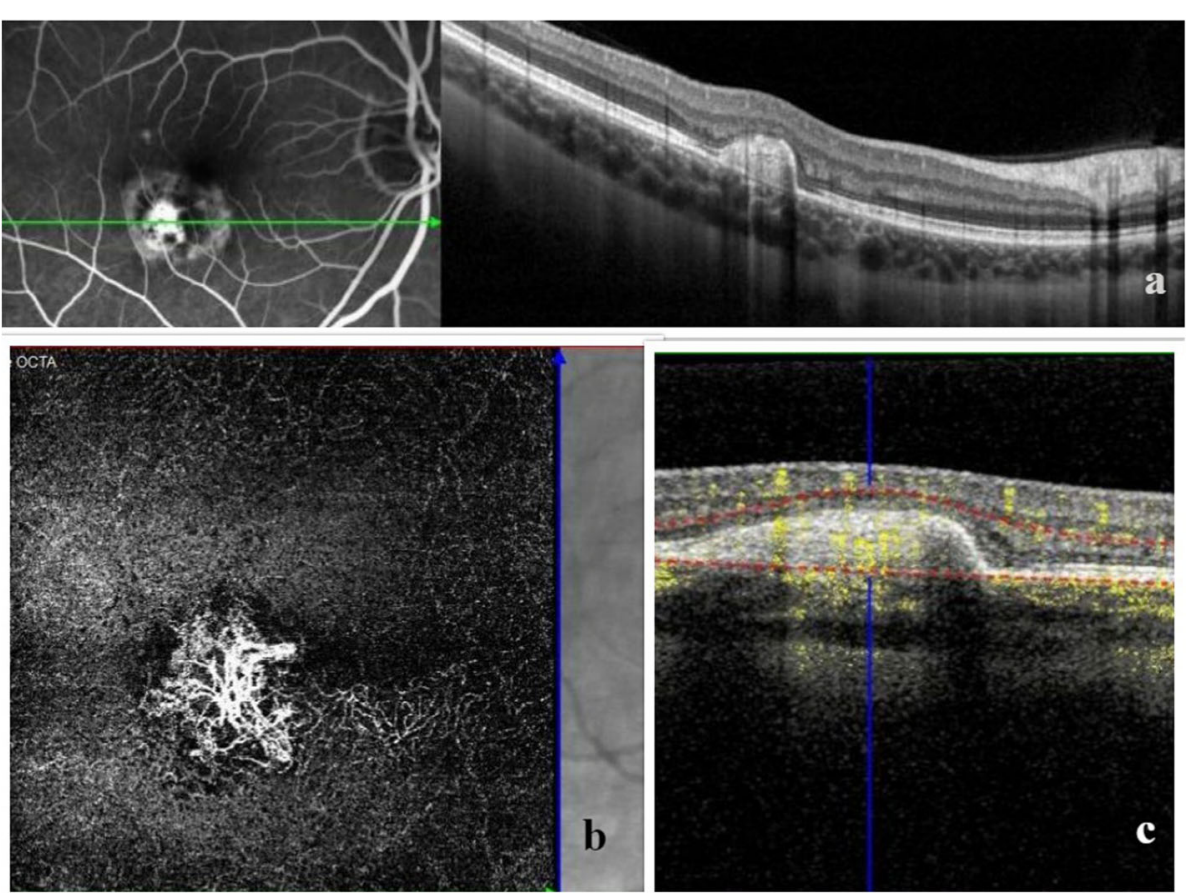

Fig. 1 FFA and structural OCT of a 36-year-old myopic woman with punctate inner choroidopathy complicated by choroidal neovascular membrane (CNV) (a). OCTA illustrates the lacy pattern of the CNV (b) associated with blood flow (yellow color) within the fibrovascular pigment epithelial detachment in the combined structural OCT/OCT-A (c)

choroiditis and the risk of its progression [80]. On FAF inactive lesions are shown hypofluorescent with sharp borders, while active lesions appear as areas of hyperfluorescence at the margin of the hypofluorescent lesions [81]. On FFA active lesions show early hypofluorescence with late hyperfluorescence and inactive ones show window defects. ICGA of inactive lesions reveals marked hypofluorescence throughout all phases of the angiogram (Fig. 2a, b). On both FFA/ICGA perfusion defects of the choriocapillaris seem to be more extensive than the RPE damage shown on FAF, which suggests that choroidal damage precedes RPE damage $[80,82]$. OCTA of choriocapillaris on active lesions shows clearly demarcated flow-deficit areas that correspond precisely to the hypofluorescent lesions on ICGA [82, 83]. Conversely, inactive lesions demonstrate some detectable flow within the areas of flow void, indicating deeper medium-to-large choroidal vessels existence and choriocapillaris loss, better detected on OCTA than on ICGA [23, 84, 85].

\section{Sarcoidosis}

In sarcoidosis, FFA is useful in revealing retinal vasculitis, while ICGA illustrates punctuate hypofluorescence [26]. OCTA may miss small granulomas with diameter smaller than the size of choriocapillaris lobules. In contrast, larger granulomas which occupy the full thickness of the choroid [86, 87], block the choriocapillaris, leading to the appearance of flow void areas on OCTA [1]. These areas co-localize with the hypofluorescent spots on ICGA, as well as with the hyporeflective choroidal lesions on enhanced depth imaging OCT (EDIOCT) $[1,23]$.

\section{Tuberculosis}

Since tuberculosis (TB) is predominantly a choroidal disease, ICGA provides useful imaging information in patients with TB posterior uveitis effectively detecting even very subtle lesions, not visible on FFA and FAF [88-90]. Partial thickness tubercular choroidal granulomas are shown as hypofluorescent lesions that become isofluorescent during the late phases, whereas full thickness ones remain hypofluorescent throughout the study [88, 91, 92]. These granulomas appear as hyporeflective lesions on EDI-OCT [89, 91]. FFA of choroidal tubercles and granulomas shows early hypofluorescence with late hyperfluorescence or isofluorescence as the deep choroidal lesions, may disappear after the early phase of the FFA due to the choriocapillary flush.

Inactive healed tubercles show transmission hyperfluorescence, while large solitary granulomas show early and progressively increasing hyperfluorescence and late pooling of dye in subretinal space of overlying exudative retinal detachment [93-95].

OCTA is able to depict these lesions as welldelineated flow void areas due to hypoperfusion of the 


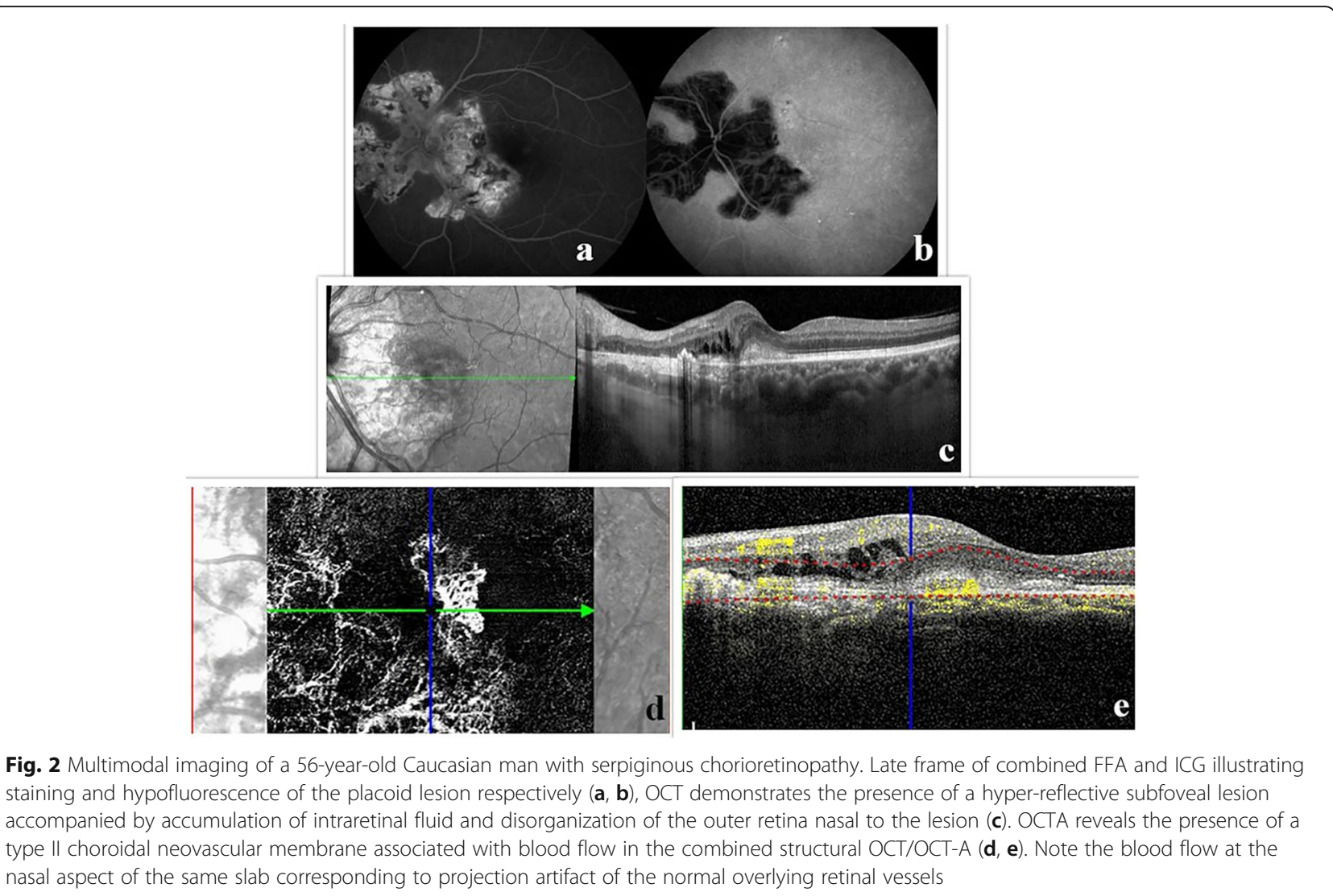

choriocapillaris, correlated well with the findings on ICGA and EDI-OCT. Few preserved islands of choriocapillaris may appear in the center of these areas. During the healing process, as a result of the choriocapillaris atrophy in some patients, medium-to-large choroidal vessels may be visualized in choriocapillaris zone on en face OCTA [89]. Vascular abnormalities, including nonneovascular tufts, tangled vessels and neovascular membranes may also be assessed with OCTA, which clearly demarcates the involvement of various retinochoroidal layers $[85,89]$.

\section{Vogt-Koyanagi-Harada}

Multimodal imaging is mandatory in order to differentiate Vogt-Koyanagi-Harada (VKH) from other clinical entities with overlapping features. OCT typically demonstrates multilobular serous macular detachments, with occasional subretinal hyper-reflective material within subretinal fluid that probably represents fibrin. In addition, using EDI-OCT Maruko et al. found increased choroidal thickness during the acute phase. FFA shows multifocal hyperfluorescent dots at the level of the RPE due to leakage or staining and late central pooling of dye in subretinal space. ICGA shows evenly distributed hypofluorescent spots (indicating active inflammation of the choroid) which may remain hypofluorescent or become isofluorescent during the late phase, depending on the depth of choroidal involvement. On OCTA, multiple dark foci are illustrated in the choriocapillaris layer, with the slab located below the RPE-Bruch's membrane complex $[1,96]$ indicating loss or severe hypoperfusion of the choriocapillaris. These foci appear as areas of flow void with discrete and sharply demarcated edges that consistently overlap with the hypofluorescent spots of the ICGA [23, 97]. This, in conjunction with no signal loss on the structural en face OCT, supports the hypothesis of true focal choriocapillaris ischemia [98, 99].

\section{Complications of uveitis}

\section{Macular edema}

Spectral-domain OCT (SD-OCT) is the imaging modality of choice for the identification and monitoring of uveitic CME as well as the evaluation of its response to treatment [100, 101].

Carrying out a quantitative OCTA analysis, Kim et al. [2] highlighted the presence of distinct areas with impaired retinal perfusion in patients with uveitic macular edema. More specifically, their data revealed remarkably decreased vascular density parameters, including skeleton and vessel density in deep retinal layer (DRL) of uveitic eyes with active CME compared with normal eyes. These alterations in deep capillary plexus (DCP) 
corresponded to the site of intraretinal cystoid spaces in inner retina, suggesting that CME may lead to displacement of retinal capillaries or even attenuation of the DRL signal (Fig. 3).

\section{Retinal ischemia}

Uveitis is frequently associated with vascular inflammation, which may lead to occlusive vasculitis characterized by retinal ischemia. Fundoscopy reveals cotton-wool spots and hemorrhages, followed by the development of telangiectatic vessels and neovascularization in more severe cases [102].

Retinal vascular occlusion is demonstrated on FFA as areas of capillary shutdown. OCTA may facilitate the evaluation of such patients by providing detailed information on ischemic areas, which present with increased intercapillary spaces on OCTA scans. The limitation, as previously mentioned, is the inability to obtain OCTA images of the peripheral retina.

\section{Retinal neovascularization}

Retinal NV may develop in uveitic patients through inflammatory and ischemic mechanisms. It is frequently seen in Behçet's disease (ABD), sarcoidosis, pars planitis, Eales disease, tuberculosis, systemic lupus erythematosus (SLE), and idiopathic retinal vasculitis [103, 104].
Although FFA is capable of demonstrating retinal NV, it cannot separately illustrate the major retinal capillary networks (superficial and deep) and may fail to reveal retinal ischemia in some of the aforementioned diseases [105]. OCTA is the imaging modality that provides detailed delineation of the microvascular structures and their potential abnormalities [105-107]. Furthermore, OCTA facilitates assessment of the precise spatial extent (depth and size) and morphological features of these intraretinal abnormalities that cannot be clearly visualized on FFA [106, 108]. Neovascular complex usually appears on OCTA as a small tuft of high-flow tiny vessels with curvilinear morphology, while abnormal retinal circulation's communications or pathological retinalretinal anastomosis can also be detected [106, 109-111]. OCTA may also be sensitive enough to reveal the extent of macular ischemia and record vascular flow changes during the course of the disease [106, 112]. However, vascular alterations in far or mid-periphery have to be determined by FFA [106].

\section{Choroidal neovascularization}

Choroidal neovascularization (CNV) is one of the main sight-threatening complications in uveitis with an incidence of $2 \%$ within uveitic population [113]. It predominantly occurs in eyes with posterior uveitis, being
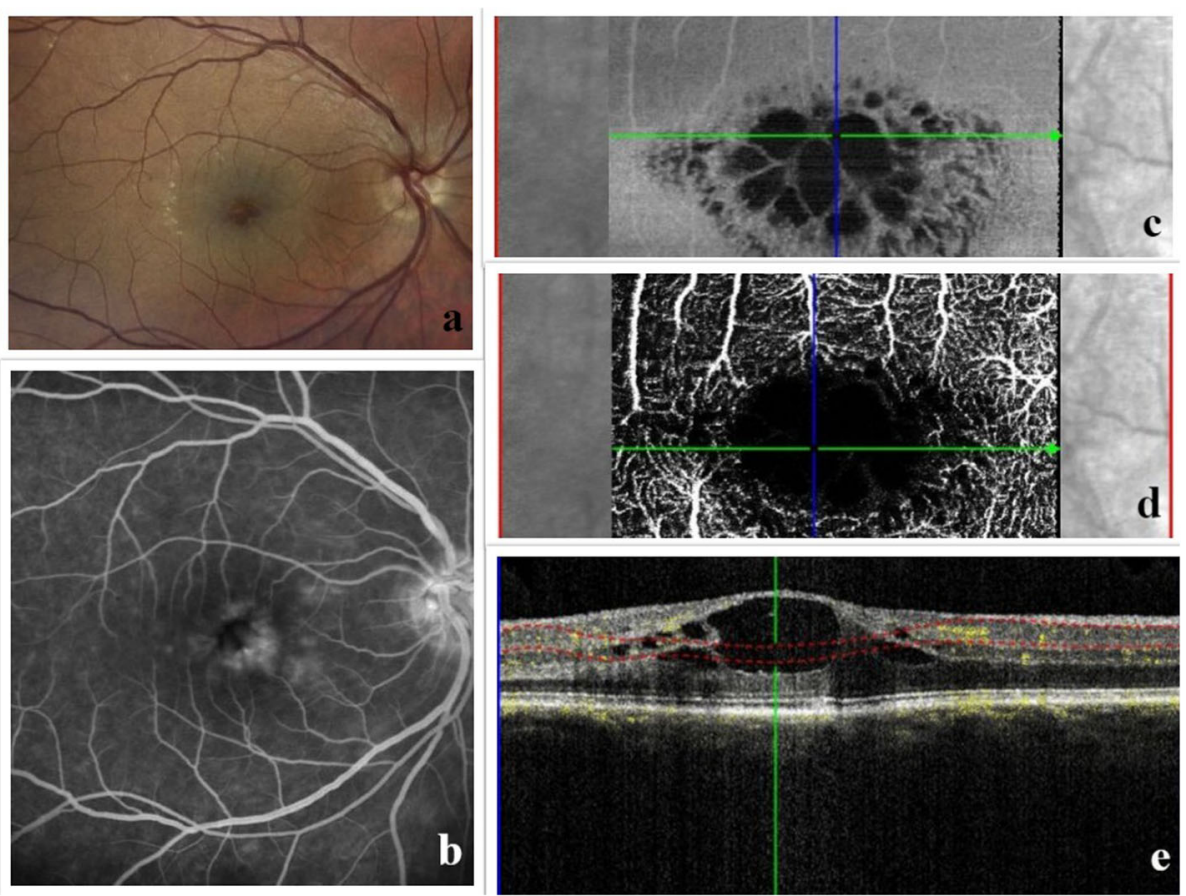

Fig. 3 Multimodal imaging of a 36-year-old Caucasian woman with intermediate uveitis. Color photo showing cystoid spaces with abnormal foveal reflex (a), FFA demonstrating petaloid pattern of fluorescein leakage along with hyperfluorescence of the optic disc (b), C-scan taken at the level of superficial vascular plexus with the corresponding en-face image exhibiting clearly visible cystoid lesions (c), the OCT-A illustrates flow void cystoid areas at the macula coupled with an enlargement of the foveal avascular zone (d), the B-scan OCT angiogram passing through the foveal depression (e) 
particularly common in $\mathrm{MCP}$, PIC, ocular histoplasmosis syndrome, and serpiginous choroiditis $[3,64-66,75,78$, 79, 114, 115] (Fig. 2c).

It has been suggested that a combination of inflammatory and associated ischemic events decompensate the previously established balance between normal angiogenetic factors and various inflammatory mediators. The choroid-produced vascular endothelial factor (VEGF) and inflammatory mediators, including interleukin-1 (IL1) and tumor necrosis factor a (TNF-a), play a role in the development of inflammatory $\mathrm{CNV}$ through the disruption of the choriocapillaris, Bruch's membrane and retinal pigment epithelium (RPE) [1, 3, 116-119].

Early diagnosis of $\mathrm{CNV}$ is crucial in order to accomplish more effective management and favorable prognosis of the disease. However, differentiating purely inflammatory lesions from inflammatory CNV can be challenging in uveitic patients [1,64, 120, 121].

OCTA may facilitate early detection of CNV, when findings of other imaging modalities are inconclusive. It has the utility of delineating a well-circumscribed neovascular network inside the area of a lesion without being obscured by dye leakage [64, 66] (Fig. 2d, e). OCTA is effective in revealing $\mathrm{CNV}$ despite existing subretinal fluid or hemorrhage, prevailing in such cases over FFA [64]. In addition, it is very efficient in monitoring the progress of $\mathrm{CNV}$ lesions that were under treatment and their response to it. Lesions that show no blood flow signal on OCTA can be distinguished from potential CNV lesions and therefore are classified as purely inflammatory one s[59]. Although it has demonstrated higher sensitivity in identifying CNV than conventional dye-based angiography, it still lacks the ability to determine which neovascular membranes are clinically active $[66,122]$. It can be speculated that $\mathrm{CNV}$ which are identified on OCTA without active leakage on FFA may represent "quiescent $\mathrm{CNV}$ " which requires a different follow-up schedule [64]. S-OCT of the OCTA has lately been proved really helpful in this direction. It allows active flow detection (which is usually colored) and, therefore, valuable information about perfusion of vessels in order to distinguish active CNV lesions [123]. Overall, OCTA alone cannot distinguish between active and inactive $\mathrm{CNVs}$ and should be integrated into a multimodal imaging approach [124]. However, it is a critical adjunct in identifying the presence of $\mathrm{CNV}$ and, therefore, a coguide in the therapeutic management and monitoring of those patients $[1,66]$.

\section{Conclusions}

Undoubtedly OCTA is gaining increasing popularity among retina specialists turning into an invaluable asset in the assessment of various retinal diseases, including uveitis. It has the potential to significantly alter the approach in diagnosis and management of uveitic entities by shedding new light into the pathophysiology of abnormal vascular changes in inflammatory conditions. Therefore, a future of a more optimal, non-invasive and valid monitoring of the uveitic patients is becoming apparent with the contribution of this imaging modality.

OCTA is able to detect and investigate a lesion and its precise spatial extent and to calculate the area and density of microvascular flow, as well as flow void areas, in a three-dimensional manner. Furthermore, it overcomes the burden of the masking effect by other elements noticed in conventional angiography. This along with the potentially future established measurements of other flow indices on OCTA, through the chorioretinal vascular plexus and through the avascular compartments, make OCTA unique in quantifying vascular perfusion and ischemia in uveitic disease. Additionally, earlier detection of a uveitic entity or its recurrence may be possible, even before the manifestation of obvious morphological changes and clinical signs.

OCTA is useful for detecting CNV with some limitations. This imaging modality lacks the ability to delineate activity of neovascular membranes. Certain areas including prevention and identification of image artifacts and projection errors require improvement.

More precise information about deeper structures may also be obtained by future use of longer wavelength monochromatic light, while the requirement of larger fields of view with higher resolution and decreased motion artifacts may be fulfilled by larger scan-patterns or faster scanning speeds. OCTA has the following limitations: inability to illustrate leakage and detect blood flow below the slowest detectable flow. Nevertheless, its non-invasive and reproducible nature can assist in a better patient compliance.

Overall, OCTA is a promising tool that continues to evolve and may diminish the need of dye-based angiograms in the future. It has been shown to have high potential to impact clinical care in uveitis however, despite its promising future, it is of note that there is limited experience in this technology, and further large scale studies are required in order to establish it as an irreplaceable clinical utility.

\footnotetext{
Abbreviations

ABD: Adamantiades-Behçet disease; APMPPE: Acute posterior multifocal placoid pigment epitheliopathy; BRC: Birdshot retinochoroiditis; CME: Cystoid macular edema; CNV: Choroidal neovascularization; DCP: Deep capillary plexus; DRL: Deep retinal layer; EDI: Enhanced depth imaging; FAF: Fundus autofluorescence; FAZ: Foveal avascular zone; FFA: Fundus fluorescein angiography; HLA: Human leukocyte antigen; ICGA: Indocyanine green angiography; IL: Interleukin; MCP: Multifocal choroiditis and panuveitis; MEWDS: Multiple evanescent white dot syndrome; MS: Multiple sclerosis; OCT: Optical coherence tomography; OCTA: Optical coherence tomography angiography; PIC: Punctate inner choroidopathy; RPE: Retinal pigment epithelium; SCP: Superficial capillary plexus; SD: Spectral domain; SLE: Systemic lupus erythematosus; TB: Tuberculosis; TB: Tuberculosis; TNF: Tumor necrosis factor; VKH: Vogt-Koyanagi-Harada
} 


\section{Acknowledgements}

Not applicable.

\section{Authors' contributions}

Authors PT, EMK, OG, and CP contributed to various sections of the manuscript. The idea was conceived by PT. The data and literature review as well as drafting of the manuscript was performed by all the authors. PT compiled all the sections from various authors. The final manuscript was read, critically reviewed, and submitted for publication by all the authors.

\section{Funding}

The research was supported by the National Institute for Health Research (NIHR) Biomedical Research Centre based at Moorfields Eye Hospital NHS Foundation Trust and UCL Institute of Ophthalmology. The views expressed are those of the author(s) and not necessarily those of the NHS, the NIHR or the Department of Health.

\section{Availability of data and materials}

Since this is a review article, there are no data repositories for this manuscript.

\section{Ethics approval and consent to participate}

Since this is a review article, there are no data repositories for this manuscript.

\section{Consent for publication}

Not applicable.

\section{Competing interests}

The authors declare that they have no competing interests.

\section{Author details}

'Vitreoretinal \& Uveitis Department, Ophthalmica Clinic, Vas.Olgas 196 and Ploutonos, 54655 Thessaloniki, Greece. ${ }^{2}$ Department of Ophthalmology, Hippokrateio General Hospital of Thessaloniki, 49 Konstantinoupoleos Street, 54642 Thessaloniki, Greece. ${ }^{3}$ Uveitis Department, Moorfields Eye Hospital, 162 City Rd, London EC1V 2PD, UK.

\section{Received: 13 December 2018 Accepted: 18 November 2019} Published online: 23 December 2019

\section{References}

1. Pichi F, Sarraf D, Arepalli S et al (2017) The application of optical coherence tomography angiography in uveitis and inflammatory eye diseases. Prog Retin Eye Res 59:178-201

2. Kim AY, Rodger DC, Shahidzadeh A et al (2016) Quantifying retinal microvascular changes in uveitis using spectral-domain optical coherence tomography angiography. Am J Ophthalmol 171:101-112

3. Agarwal A, Hassan M, Afridi R et al (2016) The role of optical coherence tomography angiography in the management of uveitis. Int Ophthalmol Clin 56(4):1-24

4. Hagag AM, Gao SS, Yali Jia DH (2017) Optical coherence tomography angiography: technical principles and clinical applications in ophthalmology. Taiwan J Ophthalmol 7:115-129

5. Novotny HR, Alvis DL (1961) A method of photographing fluorescence in circulating blood in the human retina. Circulation 24(1):82-86

6. Bursell SE, Clermont AC, Kinsley BT, Simonson DC, Aiello LM, Wolpert HA (1996) Retinal blood flow changes in patients with insulin-dependent diabetes mellitus and no diabetic retinopathy. Invest Ophthalmol Vis Sci 37(5):886-897

7. Pece A, Sannace C, Menchini U et al (2005) Fluorescein angiography and indocyanine green angiography for identifying occult choroidal neovascularization in age-related macular degeneration. Eur J Ophthalmol 15(6):759-763

8. Rabkin MD, Bellhorn MB, Bellhorn RW (1977) Selected molecular weight dextrans for in vivo permeability studies of rat retinal vascular disease. Exp Eye Res Jun 24(6):607-612

9. Kwiterovich KA, Maguire MG, Murphy RP et al (1991) Frequency of adverse systemic reactions after fluorescein angiography: results of a prospective study. Ophthalmol 98(7):1139-1142
10. Yannuzzi LA, Rohrer KT, Tindel LJ et al (1986) Fluorescein angiography complication survey. Ophthalmol 93(5):611-617

11. Yannuzzi LA, Slakter JS, Sorenson JA, Guyer DR, Orlock DA (1992) Digital indocyanine green videoangiography and choroidal neovascularization. Retina 12(3):191-223

12. Reichel E, Pollock DA, Duker JS, Puliafito CA (1995) Indocyanine green angiography for recurrent choroidal neovascularization in age-related macular degeneration. Ophthalmic Surg Lasers 26(6):513-518

13. Guyer DR, Yannuzzi LA, Slakter JS, Sorenson JA, Hope-Ross M, Orlock DR (1994) Digital indocyanine-green videoangiography of occult choroidal neovascularization. Ophthalmol 101(10):1727-1737

14. Reichel E, Duker JS, Puliafito CA (1995) Indocyanine green angiography and choroidal neovascularization obscured by hemorrhage. Ophthalmol 102(12): 1871-1876

15. Hope-Ross M, Yannuzzi LA, Gragoudas ES et al (1994) Adverse reactions due to indocyanine green. Ophthalmol 101(3):529-533

16. Ho AC, Yannuzzi LA, Guyer DR, Slakter JS, Sorenson JA, Orlock DA (1994) Intraretinal leakage of indocyanine green dye. Ophthalmol 101(3):534-541

17. Benya R, Quintana J, Brundage B (1989) Adverse reactions to indocyanine green: a case report and a review of the literature. Cathet Cardiovasc Diagn 17(4):231-233

18. Kashani AH, Chen CL, Gahm JK et al (2017) Optical coherence tomography angiography: a comprehensive review of current methods and clinical applications. Prog Retin Eye Res 60:66-100

19. Kraus MF, Potsaid B, Mayer MA et al (2012) Motion correction in optical coherence tomography volumes on a per A-scan basis using orthogonal scan patterns. Biomed Opt Express 3(6):1182

20. Huang D, Jia Y, Gao SS, Lumbroso B, Rispoli M (2016) Optical coherence tomography angiography using the Optovue device. Dev Ophthalmol 56:6-12

21. Rosenfeld PJ, Durbin MK, Roisman L et al (2016) ZEISS Angioplex ${ }^{\text {TM }}$ spectral $^{2}$ domain optical coherence tomography angiography: technical aspects. Dev Ophthalmol 56:18-29

22. Liu L, Gao SS, Bailey ST, Huang D, Li D, Jia Y (2015) Automated choroidal neovascularization detection algorithm for optical coherence tomography angiography. Biomed Opt Express 6(9):3564

23. Pichi F, Sarraf D, Morara M, Mazumdar S, Neri P, Gupta V (2017) Pearls and pitfalls of optical coherence tomography angiography in the multimodal evaluation of uveitis. J Ophthalm Inflamm Infect 7(1):20

24. Pichi F, Carrai P, Srivastava SK, Lowder CY, Nucci P, Neri P (2016) Genetic of uveitis. Int Ophthalmol 36(3):419-433

25. Kotsolis Al, Killian FA, Ladas ID, Yannuzzi LA (2010) Fluorescein angiography and optical coherence tomography concordance for choroidal neovascularisation in multifocal choroidtis. Br J Ophthalmol 94(11):1506-1508

26. Agrawal R, Biswas J, Gunasekaran D (2013) Indocyanine green angiography in posterior uveitis. Indian J Ophthalmol 61(4):148

27. Dzurinko VL, Gurwood AS, Price JR (2004) Intravenous and indocyanine green angiography. Optometry 75(12):743-755

28. Herbort CP, Mantovani A, Papadia M (2012) Use of indocyanine green angiography in uveitis. Int Ophthalmol Clin 52(4):13-31

29. Leder HA, Campbell JP, Sepah YJ et al (2013) Ultra-wide-field retinal imaging in the management of non-infectious retinal vasculitis. J Ophthalmic Inflamm Infect 3(1):30

30. Lee J, Rosen R (2016) Optical coherence tomography angiography in diabetes. Curr Diab Rep 16(12):123

31. Cunha-Vaz J (1979) The blood-ocular barriers. Surv Ophthalmol 23(5): 279-296

32. Raviola G (1977) The structural basis of the blood-ocular barriers. Exp Eye Res 25:27-63

33. Khairallah M, Abroug N, Khochtali S et al (2017) Optical coherence tomography angiography in patients with Behçet uveitis. Retina 37(9): 1678-1691

34. Takase N, Nozaki M, Kato A, Ozeki H, Yoshida M, Ogura Y (2015) Enlargement of foveal avascular zone in diabetic eyes evaluated by en face optical coherence tomography angiography. Retina 35(11):2377-2383

35. Scarinci F, Nesper PL, Fawzi AA (2016) Deep retinal capillary nonperfusion is associated with photoreceptor disruption in diabetic macular ischemia. Am J Ophthalmol 168:129-138

36. Couturier A, Mané V, Bonnin S et al (2015) Capillary plexus anomalies in diabetic retinopathy on optical coherence tomography angiography. Retina 35(11):2384-2391 
37. Nobre Cardoso J, Keane PA, Sim DA et al (2016) Systematic evaluation of optical coherence tomography angiography in retinal vein occlusion. Am J Ophthalmol. 163:93-107e6

38. Sanfilippo CJ, Klufas MA, Sarraf D, Tsui I (2015) Optical coherence tomography angigraphy of sickle cell maculopathy. Retin Cases Brief Rep 9(4):360-362

39. Herbort CP Jr, Pavesio C, LeHoang P et al (2017) Why birdshot retinochoroiditis should rather be called 'HLA-A29 uveitis'? Br J Ophthalmol 101(7):851-855

40. de Carlo TE, Bonini Filho MA, Adhi M, Duker JS (2015) Retinal and choroidal vasculature in birdshot chorioretinopathy analyzed using spectral domain optical coherence tomography angiography. Retina 35(11):2392-2399

41. Pohlmann D, Macedo S, Stübiger N, Pleyer U, Joussen AM, Winterhalter S (2017) Multimodal imaging in birdshot retinochoroiditis. Ocul Immunol Inflamm Oct 25(5):621-632

42. Spaide RF (2015) Volume rendering of optical coherence tomography angiography reveals extensive retinal vascular contributions to neovascularization in ocular toxoplasmosis. Retina 35(11):2421-2422

43. Moschos MM, Gouliopoulos NS, Kalogeropoulos C (2014) Electrophysiological examination in uveitis: a review of the literature. Clin Ophthalmol 8:199-214

44. Dell'Omo R, Pavesio CE (2012) Multiple evanescent white dot syndrome (MEWDS). Int Ophthalmol Clin 52(4):221-228

45. Dell'Omo R, Wong R, Marino M, Konstantopoulou K, Pavesio C (2010) Relationship between different fluorescein and indocyanine green angiography features in multiple evanescent white dot syndrome. Br J Ophthalmol 94(1):59-63

46. Chang AA, Zhu M, Billson F (2005) The interaction of indocyanine green with human retinal pigment epithelium. Invest Ophthalmol Vis Sci 46(4): 1463-1467

47. Pichi F, Srvivastava SK, Chexal S et al (2016) En face optical coherence tomography and optical coherence tomography angiography of multiple evanescent white dot syndrome: new Insights Into Pathogenesis. Retina 1 : 178-188

48. Labriola LT, Legarreta AD, Legarreta JE et al (2016) Imaging with multimodal adaptive-optics optical coherence tomography in multiple evanescent white dot syndrome: the structure and functional relationship. Retin Cases Brief Rep 10(4):302-309

49. Yannuzzi NA, Swaminathan SS, Zheng F et al (2017) Swept-source OCT angiography shows sparing of the choriocapillaris in multiple evanescent white dot syndrome. Ophthalmic Surg Lasers Imaging Retina 48(1):69-74

50. Quillen DA, Davis JB, Gottlieb JL et al (2004) The white dot syndromes. Am J Ophthalmol 137(3):538-550

51. Dhaliwal RS, Maguire AM, Flower RW, Arribas NP (1993) Acute posterior multifocal placoid pigment epitheliopathy: an indocyanine green angiographic study. Retina 13(4):317-325

52. Mrejen S, Sarraf D, Chexal S, Wald K, Freund KB (2016) Choroidal involvement in acute posterior multifocal placoid pigment epitheliopathy. Ophthalmic Surg Lasers Imaging Retina 47(1):20-26

53. Spaide RF, Yannuzzi LA, Slakter J (1991) Choroidal vasculitis in acute posterior multifocal placoid pigment epitheliopathy. Br J Ophthalmol 75(11): 685-687

54. Deutman, AF, Boen-Tan TN. Oosterhuis, JA (1973) Proceedings: acute posterior multifocal placoid pigment epitheliopathy. Ophthalmologica 167 368-372.

55. Deutman AF, Oosterhuis JA, Boen-Tan TN, Aan de Kerk AL (1972) Acute posterior multifocal placoid pigment epitheliopathy. Pigment epitheliopathy of choriocapillaritis? Br J Ophthalmol 56(12):863-874

56. Yuzawa M, Kawamura A, Matsui M (1994) Indocyanine green video angiographic findings in acute posterior multifocal placoid pigment epitheliopathy. Acta Ophthalmol 72(1):128-133

57. Park D, Schatz H, McDonald HR, Johnson RN (1995) Indocyanine green angiography of acute multifocal posterior placoid pigment epitheliopathy. ophthalmology 102(12):1877-1883.

58. Dolz-Marco R, Rodríguez-Ratín Á, Hernández-Martínez P, Pascual-Camps I, Andreu-Fenoll M, Gallego-Pinazo R (2014) Macular retinal and choroidal thickness in unilateral relentless placoid chorioretinitis analyzed by sweptsource optical coherence tomography. J Ophthalmic Inflamm Infect 4(1):1-6

59. Klufas MA, Phasukkijwatana N, lafe NA et al (2017) Optical coherence tomography angiography reveals choriocapillaris flow reduction in placoid chorioretinitis. Ophthalmol Retin 1(1):77-91
60. Kinouchi R, Nishikawa N, Ishibazawa A, Yoshida A (2017) Vascular rarefaction at the choriocapillaris in acute posterior multifocal placoid pigment epitheliopathy viewed on OCT angiography. Int Ophthalmol 37(3):733-736

61. Choi W, Mohler KJ, Potsaid B et al (2013) Choriocapillaris and choroidal microvasculature imaging with ultrahigh speed OCT angiography. PLoS One 8(12):e81499

62. Ferrara D, Waheed NK, Duker JS (2016) Investigating the choriocapillaris and choroidal vasculature with new optical coherence tomography technologies. Prog Retin Eye Res 52:130-155

63. Heiferman MJ, Rahmani S, Jampol LM et al (2017) Acute posterior multifocal placoid pigment epitheliopathy on optical coherence tomography angiography. Retina 37(11):2084-2094

64. Cheng L, Chen X, Weng S et al (2016) Spectral-domain optical coherence tomography angiography findings in multifocal choroiditis with active lesions. Am J Ophthalmol 169:145-161

65. Thorne JE, Wittenberg S, Jabs DA et al (2006) Multifocal choroiditis with panuveitis. Incidence of ocular complications and of loss of visual acuity. Ophthalmol 113(12):2310-2316

66. Levison AL, Baynes KM, Lowder CY, Kaiser PK, Srivastava SK (2017) Choroidal neovascularisation on optical coherence tomography angiography in punctate inner choroidopathy and multifocal choroiditis. Br J Ophthalmol 101(5):616-622

67. Brown J Jr, Folk JC, Reddy CV, Kimura AE (1996) Visual prognosis of multifocal choroiditis, punctate inner choroidopathy, and the diffuse subretinal fibrosis syndrome. Ophthalmology 103(7):1100-1105

68. Parnell JR, Jampol LM, Yannuzzi LA, Gass JD, Tittl MK (2001) Differentiation between presumed ocular histoplasmosis syndrome and multifocal choroiditis with panuveitis based on morphology of photographed fundus lesions and fluorescein angiography. Arch Ophthalmol 119(2):208-212

69. Shakoor A, Vitale AT (2012) Imaging in the diagnosis and management of multifocal choroiditis and punctate inner choroidopathy. Int Ophthalmol Clin 52(4):243-256

70. Onal S, Tugal-Tutkun I, Neri P, P Herbort C (2014) Optical coherence tomography imaging in uveitis. Int Ophthalmol 34(2):401-435.

71. Spaide RF, Goldberg N, Freund KB (2013) Redefining multifocal choroiditis and panuveitis and punctate inner choroidopathy through multimodal imaging. Retina 33:1315

72. Vance SK, Khan S, Klancnik JM, Freund KB (2011) Characteristic spectraldomain optical coherence tomography findings of multifocal choroiditis. Retina 31(4):717-723

73. Cerquaglia A, Lupidi M, Fiore T, laccheri B, Perri P, Cagini C (2017) Deep inside multifocal choroiditis: an optical coherence tomography angiography approach. Int Ophthalmol 37(4):1047-1051

74. Baumal CR, De Carlo TE, Waheed NK, Salz DA, Witkin AJ, Duker JS (2015) Sequential optical coherence tomographic angiography for diagnosis and treatment of choroidal neovascularization in multifocal choroiditis. JAMA Ophthalmol 133(9):1087-1090

75. Perentes $Y$, Tran VT, Sickenberg M, Herbort CP (2005) Subretinal neovascular membranes complicating uveitis: frequency, treatments, and visual outcome. Ocul Immunol Inflamm 13(2-3):219-224

76. Patel KH, Birnbaum AD, Tessler HH, Goldstein DA (2011) Presentation and outcome of patients with punctate inner choroidopathy at a tertiary referral center. Retina 31:1387

77. Watzke RC, Packer AJ, Folk JC, Benson WE, Burgess D, Ober RR (1984) Punctate inner choroidopathy. Am J Ophthalmol 98(5):572-584

78. Read RW, Rechodouni A, Butani N et al (2001) Complications and prognostic factors in Vogt-Koyanagi-Harada disease. Am J Ophthalmol 131(5):599-606

79. Klufas MA, O'Hearn T, Sarraf D (2015) Optical coherence tomography angiography and widefield fundus autofluorescence in punctate inner choroidopathy. Retin Cases Brief Rep 9(4):323-326

80. Piccolino FC, Grosso A, Savini E (2009) Fundus autofluorescence in serpiginous choroiditis. Graefe's Arch Clin Exp Ophthalmol 247(2):179-185

81. Yeh S, Forooghian F, Wong WT et al (2010) Fundus autofluorescence imaging of the white dot syndromes. Arch Ophthalmol 128(1):46-56

82. Montorio D, Giuffrè C, Miserocchi E et al (2018) Swept-source optical coherence tomography angiography in serpiginous choroiditis. $\mathrm{Br} J$ Ophthalmol 102(7):991-995

83. Mandadi SKR, Agarwal A, Aggarwal K et al (2017) Novel findings on optical coherence tomography angiography in patients with tubercular serpiginous-like choroiditis. Retina 37(9):1647-1659 
84. Bansal R, Gupta A, Gupta V (2012) Imaging in the diagnosis and management of serpiginous choroiditis. Int Ophthalmol Clin 52(4):229-236

85. Khan HA, Shahzad MA (2017) Multimodal imaging of serpiginous choroiditis. Optom Vis Sci 94(2):265-269

86. Chen L, Xu G (2013) Extensive choroidal infiltrates in choroidal biopsy proven ocular sarcoidosis. Retin Cases Br Reports 7(1):69-70

87. Mehta H, Sim DA, Keane PA et al (2015) Structural changes of the choroid in sarcoid- and tuberculosis-related granulomatous uveitis. Eye 29(8):1060-1068

88. Wolfensberger TJ, Piguet B, Herbort CP (1999) Indocyanine green angiographic features in tuberculous chorioretinitis. Am J Ophthalmol 127(3):350-353

89. Invernizzi A, Mapelli C, Viola F et al (2015) Choroidal granulomas visualized by enhanced depth imaging optical coherence tomography. Retina 35(3): 525-531

90. Herbort CP, LeHoang P, Guex-Crosier Y (1998) Schematic interpretation of indocyanine green angiography in posterior uveitis using a standard angiographic protocol. Ophthalmology 105(3):432-440

91. Agarwal A, Mahajan S, Khairallah M, Mahendradas P, Gupta A, Gupta V (2017) Multimodal imaging in ocular tuberculosis. Ocul Immunol Inflamm 25(1):134-145

92. Milea D, Fardeau C, Lumbroso L et al (1999) Indocyanine green angiography in choroidal tuberculomas. Br J Ophthalmol 83:753

93. Gupta V, Gupta A, Rao NA (2007) Intraocular tuberculosis-an update. Surv Ophthalmol 52(6):561-587

94. Gupta V, Shoughy SS, Mahajan S et al (2015) Clinics of ocular tuberculosis. Ocul Immunol Inflamm 23(1):14-24

95. Mehta S (2006) Fundus fluorescein angiography of choroidal tubercles: case reports and review of literature. Indian J Ophthalmol 54(4):273

96. Cennamo G, Romano MR, lovino C, De Crecchio G, Cennamo G (2017) Optical coherence tomography angiography in incomplete acute VogtKoyanagi-Harada disease. Int J Ophthalmol 10(4):661-662

97. Rao NA (2007) Pathology of Vogt-Koyanagi-Harada disease. Int Ophthalmo 27(2-3):81-85

98. Aggarwal K, Agarwal A, Deokar A et al (2017) Distinguishing features of acute Vogt-Koyanagi-Harada disease and acute central serous chorioretinopathy on optical coherence tomography angiography and en face optical coherence tomography imaging. J Ophthalmic Inflamm Infect 7(1):3

99. Aggarwal K, Agarwal A, Mahajan S et al (2018) The role of optical coherence tomography angiography in the diagnosis and management of acute Vogt-Koyanagi-Harada disease. Ocul Immunol Inflamm 26(1):142-153

100. Markomichelakis NN, Halkiadakis I, Pantelia E et al (2004) Patterns of macular edema in patients with uveitis: qualitative and quantitative assessment using optical coherence tomography. Ophthalmology 111(5):946-953

101. Tran THC, de Smet MD, Bodaghi B, Fardeau C, Cassoux N, Lehoang P (2008) Uveitic macular oedema: correlation between optical coherence tomography patterns with visual acuity and fluorescein angiography. $\mathrm{Br} J$ Ophthalmol 92(7):922-927

102. Abu El-Asrar AM, Herbort CP, Tabbara KF (2009) Differential diagnosis of retinal vasculitis. Middle East Afr J Ophthalmol 16(4):202-218

103. Patel AK, Newcomb CW, Liesegang TL et al (2016) Risk of retinal neovascularization in cases of uveitis. Ophthalmology 123(3):646-654

104. Kuo IC, Cunningham ET (2000) Ocular neovascularization in patients with uveitis. Int Ophthalmol Clin 40(2):111-126

105. Yu S, Lu J, Cao D et al (2016) The role of optical coherence tomography angiography in fundus vascular abnormalities. BMC Ophthalmol 16:107

106. Chalam K, Sambhav K (2016) Optical coherence tomography angiography in retinal diseases. J Ophthalmic Vis Res 11(1):84-92

107. de Carlo TE, Romano A, Waheed NK, Duker JS (2015) A review of optical coherence tomography angiography (OCTA). Int J Retin Vitr 1(1):5

108. Lang GE, Enders C, Werner JU (2016) New possibilities in retinal diagnostics using OCT angiography. Klin Monbl Augenheilkd 233(5):613-621

109. De Carlo TE, Chin AT, Bonini Filho MA et al (2015) Detection of microvascular changes in eyes of patients with diabetes but not clinical diabetic retinopathy using optical coherence tomography angiography. Retina 35(11):2364-2370

110. Kuehlewein L, Dansingani KK, de Carlo TE et al (2015) Optical coherence tomography angiography of type 3 neovascularization secondary to agerelated macular degeneration. Retina 35(11):2229-2235

111. Miere A, Querques G, Semoun O, El Ameen A, Capuano V, Souied EH (2015) Optical coherence tomography angiography in early type 3 neovascularization. Retina. 35(11):2236-2241
112. Bonini Filho MA, Adhi M, De Carlo TE et al (2015) Optical coherence tomography angiography in retinal artery occlusion. Retina 35(11):23392346

113. Baxter SL, Pistilli M, Pujari SS et al (2013) Risk of choroidal neovascularization among the uveitides. Am J Ophthalmol 156(3):468-477

114. Patel KH, Birnbaum AD, Tessler HH, Goldstein DA (2011) Presentation and outcome of patients with punctate inner choroidopathy at a tertiary referral center. Retina 31(7):1387-1391

115. Wu K, Zhang X, Su Y et al (2016) Clinical characteristics of inflammatory choroidal neovascularization in a Chinese population. Ocul Immunol Inflamm 24(3):261-267

116. Espinosa-Heidmann DG, Suner IJ, Hernandez EP, Monroy D, Csaky KG, Cousins SW (2003) Macrophage depletion diminishes lesion size and severity in experimental choroidal neovascularization. Investig Ophthalmol Vis Sci 44(8):3586-3592

117. Dhingra N, Kelly S, Majid M a, Bailey CB, Dick AD (2010) Inflammatory choroidal neovascular membrane in posterior uveitis-pathogenesis and treatment. Indian J Ophthalmol 58(1):3-10.

118. Crane IJ, Wallace CA, McKillop-Smith S, Forrester JV (2000) CXCR4 receptor expression on human retinal pigment epithelial cells from the blood-retina barrier leads to chemokine secretion and migration in response to stromal cell-derived factor 1 alpha. J Immunol 165(8):4372-4378

119. Cha H-S, Bae E-K, Koh J-H et al (2007) Tumor necrosis factor-alpha induces vascular endothelial growth factor-C expression in rheumatoid synoviocytes. J Rheumatol 34(1):16-19

120. Kotsolis Al, Killian FA, Ladas ID, Yannuzzi LA (2010) Fluorescein angiography and optical coherence tomography concordance for choroidal neovascularisation in multifocal choroiditis. Br J Ophthalmol 94(11):1506-1508

121. Spaide RF, Klancnik JM, Cooney MJ (2015) Retinal vascular layers imaged by fluorescein angiography and optical coherence tomography angiography. JAMA Ophthalmol 133(1):45

122. Zahid S, Chen KC, Jung JJ et al (2017) Optical coherence tomography angiography of chorioretinal lesions due to idiopathic multifocal choroiditis. Retina 37(8):1451-1463

123. Jia Y, Bailey ST, Wilson DJ, et al (2014) Quantitative optical coherence tomography angiography of choroidal neovascularization in age-related macular degeneration. ophthalmology 121(7):1435-1444.

124. Astroz P, Miere A, Mrejen S et al (2018) Optical coherence tomography angiography to distinguish choroidal neovascularization from macular inflammatory lesions in multifocal choroiditis. Retina Feb 38(2):299-309

\section{Publisher's Note}

Springer Nature remains neutral with regard to jurisdictional claims in published maps and institutional affiliations.

\section{Submit your manuscript to a SpringerOpen ${ }^{\circ}$ journal and benefit from:}

- Convenient online submission

- Rigorous peer review

- Open access: articles freely available online

- High visibility within the field

- Retaining the copyright to your article

Submit your next manuscript at $>$ springeropen.com 